\title{
Statistical analysis of excitation energies in the actinide and rare-earth nuclei
}

\author{
A.I. Levon 7 A.G. Magner $]^{\oplus}$ and S.V. Radionov \\ Institute for Nuclear Research, 03680 Kyiv, Ukraine
}

(Dated: July, 1st, 2017)

\begin{abstract}
Statistical analysis of distributions of the collective states in the actinide and rare-earth nuclei is performed in terms of the nearest neighbor spacing distribution (NNSD). Several approximations, such as the linear approach to the level repulsion density and that suggested by Brody to the NNSDs were applied for the analysis. We found an intermediate character of the experimental spectra between the order and the chaos for a number of the rare-earth and actinide nuclei. They are more close to the Wigner distribution for energies limited by $3 \mathrm{MeV}$, and to the Poisson distribution for data including higher excitation energies and higher spins. The latter is in agreement with the theoretical calculations. These features are confirmed by the cumulative distributions, where the Wigner contribution dominates at smaller spacings while the Poisson one is more important at larger spacings.
\end{abstract}

\section{INTRODUCTION}

The microscopic many-body interaction of particles of the systems, such as heavy deformed nuclei, is rather complicated. Therefore, theoretical approaches to the description of nuclear excitations are helpful for understanding the properties of the collective motion in such nuclei. As the simplest approaches, one can mention calculations within the phenomenological interacting-boson model [1] and a more microscopic quasiparticle-phonon model [2]. Toward the microscopic picture, other approaches are described in Refs. [3-5]. However, one can significantly simplify the realistic many-body problem and enrich its understanding by using the nuclear models which are based on the statistical properties of the distributions of discrete levels.

Different statistical methods have been proposed to obtain information on the chaoticity versus regularity in quantum spectra of a nuclear many-body system [69], see also the well known work by Bohigas, Giannoni and Schmit [11]. The short-range fluctuation properties in experimental spectra can be analyzed in terms of the nearest-neighbor spacing distribution (NNSD) statistics. The uncorrelated sequence of energy levels, originated by a regular dynamics, is described by the Poisson distribution. In the case of a completely chaotic dynamics, the energy intervals between levels follow mainly the Wigner (Gaussian orthogonal-ensemble) distribution. An intermediate degree of chaos in energy spectra is usually obtained through a comparison of the experimental NNSDs with well known distributions [12 15] based on the fundamental works [11, 16 18]. This comparison is carried out [19 23] by using the least square-fit technique. The estimated values of parameters of these distributions shed the light on the statistical situation with considered spectra. Berry and Robnik [14] derived the NNSD starting from the microscopic expression for the level density through the Hamiltonian for a classical system. The Brody NNSD [13] is based on the expression for the level repulsion density that interpolates between the Poisson

\footnotetext{
* Email: levon@kinr.kiev.ua

$\dagger$ Email: magner@kinr.kiev.ua

$\ddagger$ Email:sergey.radionov@matfys.lth.se
}

and the Wigner distribution by only one parameter.

For a quantitative measure of the degree of chaoticity of the many-body dynamics, the statistical probability distribution $p(s)$ as function of spacings $s$ between the nearest neighboring levels can be derived within the general Wigner-Dyson (WD) approach based on the level repulsion density $g(s)$ (the units will be specified later) [6, 7, 16, 18],

$$
p(s) \propto g(s) \exp \left(-\int_{0}^{s} g\left(s^{\prime}\right) \mathrm{d} s^{\prime}\right) .
$$

This approach can be applied in the random matrix theory, see for instance Refs. [8, 18], and also, for systems with definite Hamiltonians [6, 7]. In any case, the order in such systems is approximately associated with the Poisson dependence of $p(s)$ [Eq. (11)] on the spacing $s$ variable, that is obviously related to a constant $g(s)$, independent of $s$. A chaoticity can be referred, mainly, to the Wigner distribution, as clearly follows from Eq. (1) for $g(s) \propto s$.

For a further study of the order-chaos properties of nuclear systems, it might be worth to apply a simple analytical approximation to the WD NNSD (1), keeping the link with a level repulsion density $g(s)$ [6, 7, 18]. For analysis of the statistical properties in terms of the Poisson and Wigner distributions, one can use the linear WD (LWD) approximation to the level repulsion density $g(s)$ [24]. It is the two-parameter approach; in contrast, e.g., to the one-parameter Brody approach. However, the LWD approximation, as based on a smooth analytical (linear) function $g(s)$ of $s$, can be founded within the WD theory (see Refs. [7, 24] and Appendix). Moreover, it gives a proper information on the separate Poisson order-like and Wigner chaos-like contributions.

In the present work, the two different approaches, such as the LWD approximation to the NNSD (11) of the WD theory, and the traditional Brody method are used for the statistical description of the collective-excitation energies in deformed actinide and rare-earth nuclei. This is alternative problem to that for the nuclear states of another nature; see, e.g., Refs. [10, 23]. The statistical properties of the nuclear collective states are discussed in relation to the degree of chaoticity in terms of the Poisson and Wigner distribution contributions. The main purpose is to describe these excitations in deformed nu- 
clei by using the NNSDs, in contrast to the states which can be considered as statistically excited ones in a heated system. In addition, the cumulative NNSDs show the statistical properties of these collective states as functions of the spacing variable in relation to the same limits.

This article is organized in the following way. Section III is devoted to the description of experimental data, their completeness and the unfolding procedure for calculations of the NNSD. In Section III, we present several analytical approximations to the NNSD within the Wigner-Dyson theory. The NNSD using the linear approximation to the level repulsion density, and the Brody approach as well as the cumulative distribution method (Sec. III) are compared with the experimental data in Sec. IV. Our results are summarized in Sec. V] Some details of our derivations are given in Appendix.

\section{EXPERIMENTAL NNSD}

\section{A. Experimental data}

To perform statistical analysis of the energy spacings, one needs the complete and pure sequences of levels. The completeness means no missing and no misassigned levels in the desired energy interval of the level sequence. The problem of missing levels in spectral statistics was considered for the first time by Bohigas and Pato 25] and reviewed by Gomez et al. [10]. For nuclear physics, the requirement of purity is that the levels with the same angular-momentum and parity quantum numbers should be considered in a given interval. The additional quantum numbers can be taken into account in some cases. Shriner et al. 19 21 achieved these purposes by limiting the energy interval to have well-defined, at that time, spins and parities. As a consequence, the number of selected levels with a particular spin and parity in each nucleus was usually limited to $5-8$. As shown in Table [I much longer sequences of levels [27-36] are analyzed in the present study, what is important for a statistical accuracy of their fitting procedure.

Such sequences are available at excitations of nuclei in the two-neutron transfer reactions. Most of the studies using these $(\mathrm{p}, \mathrm{t})$ reactions are devoted to the investigation of the nature of $0^{+}$states. In such reactions, one can indeed observe long sequences of the collective $0^{+}$ states. The first observation of multiple excitations with the zero angular-momentum transfer was realized for the $(\mathrm{p}, \mathrm{t})$ reaction in the odd nucleus ${ }^{229} \mathrm{~Pa}$ 26]. Such studies were undertaken later by many collaborations, e.g., in the deformed even-even actinide [27-30] and rare-earth 31-34 nuclei. Typical spectra of $0^{+}$states are shown in Fig. 11 However, the use of the $(\mathrm{p}, \mathrm{t})$ reaction is not limited only to the observation of $0^{+}$states. Long sequences of the states with higher angular momenta $2^{+}, 4^{+}$, and even $6^{+}$, along with the $0^{+}$states, were identified for nuclei in the actinide region [27 29, 35, 36]. They can be used in the statistical analysis, too. As emphasized above, the purity of all these sequences of states is maintained by the fact that all these states are collective by
TABLE I. Number of levels included in the analysis.

\begin{tabular}{cccccc}
\hline \hline & & & & & \\
Nuclei & $0^{+}$ & $2^{+}$ & $4^{+}$ & $6^{+}$ & Total \\
\hline${ }^{228} \mathrm{Th}$ & 16 & 32 & 21 & 9 & 78 \\
${ }^{230} \mathrm{Th}$ & 20 & 68 & 46 & 19 & 153 \\
${ }^{232} \mathrm{U}$ & 13 & 46 & 33 & 19 & 111 \\
${ }^{240} \mathrm{Pu}$ & 17 & 37 & 30 & 11 & 95 \\
Rare Earths $\leq 3 \mathrm{MeV}$ & 128 & & & & 128 \\
${ }^{158} \mathrm{Gd}^{168} \mathrm{Er} \leq 4 \mathrm{MeV}$ & 58 & & & & 58 \\
\hline \hline
\end{tabular}

their nature. This follows from an analysis within, e.g., the framework of the interacting boson model, and the quasiparticle phonon model [27 29, 32, 37].

Excitations of the $0^{+}$states have an advantage over those of other states. Even the weakly excited $0^{+}$states, even in the complicate and dense excitation spectra, are easily identified via angular distributions of the cross section in (p,t) reactions. Shapes of these angular distributions are mainly independent of specific structures of the individual states as well as of the transfer configurations. A few levels included in the analysis are assigned tentatively: 4 of 78 levels of actinide, and 18 of 128 levels for rare-earth nuclei in the energy region below $3 \mathrm{MeV}$. However, all of 58 levels in the ${ }^{158} \mathrm{Gd}$ and ${ }^{168} \mathrm{Er}$ nuclei for the interval $0-4 \mathrm{MeV}$ are firmly assigned. Therefore, the spectra of $0^{+}$states measured in the $(\mathrm{p}, \mathrm{t})$ reaction can be considered as complete ones in energy intervals mentioned above, that allows to perform properly the statistical analysis.

Concerning higher spin measurements, one can outlook the situation with a sequence of the $2^{+}$levels in the nucleus ${ }^{230} \mathrm{Th}$, as a typical example. Before the excitation spectra in ${ }^{230} \mathrm{Th}$ were studied by means of the $(\mathrm{p}, \mathrm{t})$ reaction, the firm $2^{+}$assignments were known only for 6 levels and tentative assignments for 21 levels, mainly, as states with 1 or $2^{+}$spin values, which are denoted by $\left(1,2^{+}\right)$[38, 39]. In Ref. 27], the 66 levels with the spin $2^{+}$were identified in the $(\mathrm{p}, \mathrm{t})$ experiment. The energies of the 5 of 66 levels, within the limit of errors, coincide with those of $\left(1,2^{+}\right)$known earlier. Moreover, five of previously known level assignments have been changed 27] with other quantum numbers. The remaining eleven levels with a tentative assignment as $\left(1,2^{+}\right)$were not observed in the $(p, t)$ reaction because, probably, they have the spin 1. Such states are not observed, practically, in the $(\mathrm{p}, \mathrm{t})$ reaction. Another reason is that these states are not collective. We emphasize once more that a collectivity is the additional condition for selections of the level sequences. Therefore, the 66 levels were analyzed in the present work. Nevertheless, let us assume that, for completeness, some of eleven levels should be included in the sequence. Then, one finds a shift of the NNSD to the Poisson distribution, which is additional to that discussed in Sec. IV.

In the case of spin $4^{+}, 30 \%$ of levels are assigned as tentative results. Their angular distributions exclude the reliable assignments of $0^{+}$and $2^{+}$spins. In addition, the 


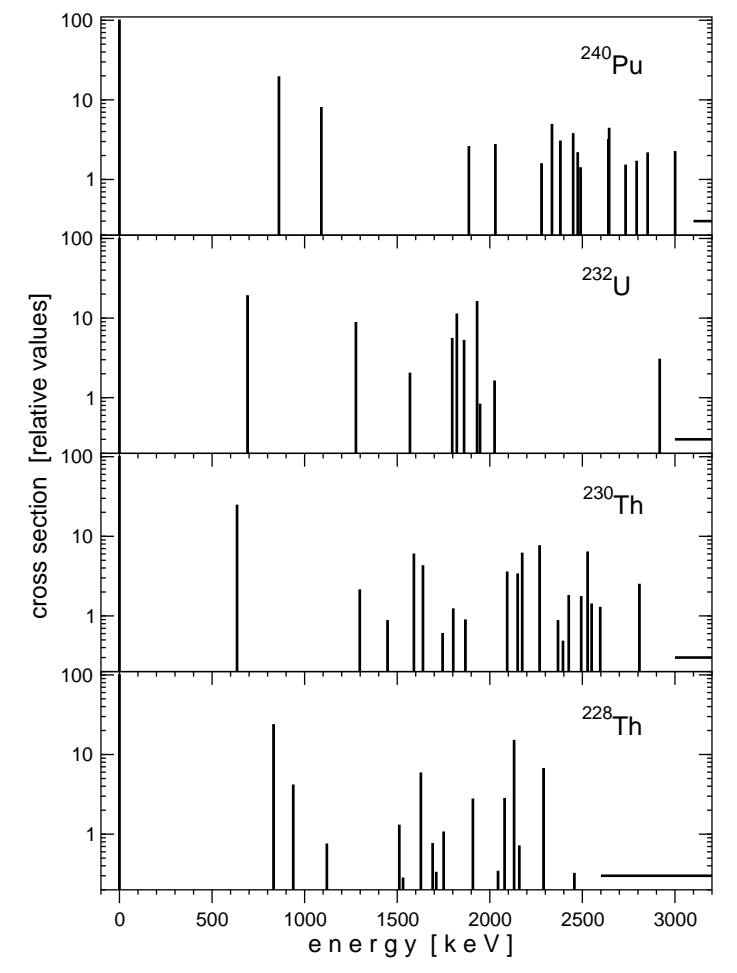

FIG. 1. The location and the $(\mathrm{p}, \mathrm{t})$ strength of $0^{+}$states in ${ }^{228} \mathrm{Th},{ }^{230} \mathrm{Th},{ }^{232} \mathrm{U}$ and ${ }^{240} \mathrm{Pu}$. Horizontal lines indicate limitations in the investigation energy.

$(\mathrm{p}, \mathrm{t})$ cross section for higher spins decreases by almost one order of the magnitude. Therefore, for these levels, one can accept the $4^{+}$spin value. Thus, all the 66 levels with the spin $4^{+}$were included into our analysis.

Notice that many of $6^{+}$levels are missing because of a sharp decrease of the $(\mathrm{p}, \mathrm{t})$ cross sections for a so large angular momentum. This is particularly true for the states that correspond to small values of $s$ : Weak peaks in a very complex and dense spectrum can be hidden in the tails of stronger neighbors. As a result, the sequence of $6^{+}$levels occurs to be not complete. The effect of missing levels in the case of $6^{+}$levels is properly discussed in Sec. IV]

\section{B. Unfolding procedure}

To compare properly the statistical properties of different sequences to each other, one should convert any set of the energy levels into a set of the normalized spacing, that can be done through the so-called unfolding procedure [40]. In this procedure, an original set of the level energies $E_{i}, i=1,2, \ldots$, is transformed into a new set $\varepsilon_{i}$ as a mapping

$$
\varepsilon_{i}=\tilde{N}\left(E_{i}\right),
$$

where $\tilde{N}(E)$ is a smooth part of the cumulative level density, $N(E)=\int_{0}^{E} d E^{\prime}\left(\mathrm{d} N / \mathrm{d} E^{\prime}\right)$, where $\mathrm{d} N / \mathrm{d} E$ is the level density. The cumulative density $N(E)$ is a staircase function that counts the number of states with energies

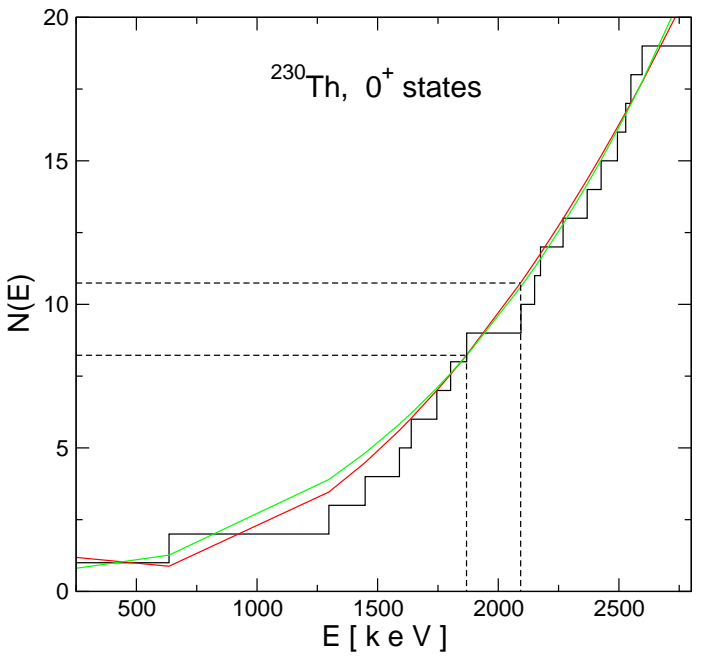

FIG. 2. Histogram of the cumulative number of states, $N(E)$, for the $0^{+}$energy spacings in ${ }^{230} \mathrm{Th}$ and its fitting by two smooth polynomials, Eq. (5), present the method of an extraction of the normalized effective NNSD from the experimental data.

$E$, which are less or equal to a given value of $E$. The decomposition of this density (or, the level density itself) into a smooth and fluctuating part is not obvious. Usually, one can use polynomial fits to a smooth part.

In what follows, the spectra will be analyzed in terms of the spacings between the unfolded energy levels (2),

$$
s_{i}=\varepsilon_{i+1}-\varepsilon_{i} .
$$

By Taylor expansion of $\tilde{N}\left(E_{i}\right)$ in Eq. (2) up to linear terms in $E_{i+1}-E_{i}$, one writes

$$
\varepsilon_{i+1}-\varepsilon_{i} \approx\left(E_{i+1}-E_{i}\right) \frac{\mathrm{d} \widetilde{N}\left(E_{i}\right)}{\mathrm{d} E_{i}}=\frac{E_{i+1}-E_{i}}{D_{i}},
$$

where $D_{i}=1 /\left[\mathrm{d} \tilde{N}\left(E_{i}\right) / \mathrm{d} E_{i}\right]$ is the average level spacing locally in a small vicinity of $E_{i}$. For the approximation (4), one assumes that the average dimensionless spacing between the unfolded levels (2) is one, provided that the smooth level density $\mathrm{d} \widetilde{N}\left(E_{i}\right) / \mathrm{d} E_{i}$ is a slowly varying function of the energy $E_{i}$.

Thus, for each observed level, the value of the fit function $\widetilde{N}(E)$ can be used for the generation of a spacing distribution, as illustrated in Fig. 2. As one can see, a small (large) energy spacing corresponds to a small (large) spacing in $\widetilde{N}(E)$, according to the monotonic mapping [Eqs. (3) and (2)]. The distribution $\widetilde{N}(E)$ was then used for building the final NNSDs. Since the experimental data for a particular sequence is statistically limited we compiled the distribution for all sequences to get the entire unified set of the nearest neighbor spacing distribution with relatively a small sampling interval $\gamma_{s}$ (see Sec. IV and Appendix).

As shown in Fig. 1 the spectrum of states is sparse and widely spread. Therefore, the polynomial fitting looks as a difficult problem. Indeed, using different polynomials to fit the full spectrum of states, one obtains a 
different fit at the beginning of the spectrum. As a result, one finds somewhat a different NNSD. It is caused by a specific property of the energy spectra: The spacing between the two lowest levels is much larger than that between all other levels. In the statistical analysis, the first energy interval contributes to the NNSD in the region of too large $s$. Therefore, this level can be discarded without any completeness violation. Then, the remaining smooth-state trend is well reproduced by any low-order polynomial, and various polynomials lead to a very close NNSDs. In the analysis we used the following polynomials:

$$
\tilde{N}=a_{0}+a_{1} E+a_{2} E^{2}, \quad \tilde{N}=a_{0}+a_{1} E^{2}+a_{2} E^{4},
$$

where $a_{i}$ are fitting parameters. Absolute values of $s_{i}$ for each level, obtained with different polynomials, are distinguished somehow. But the final result for spacing distributions differs only by the part with a low statistics. Therefore, the NNSD results based on both polynomials are stable and compiled well in our calculations.

Note that another unfolding procedure [41] using an empirical formula $\tilde{N}(E)=\exp \left[\left(E-E_{0}\right) / T\right]+N_{0}$, where $T, E_{0}$, and $N_{0}$ are the fitting parameters, was applied [19] for the statistical analysis. As was shown [23] for excited states in the spherical nucleus ${ }^{208} \mathrm{~Pb}$, both procedures yield approximately similar results for NNSDs. We found that the polynomial empiric functions $\widetilde{N}(E)$ [Eq. (5)] are more suitable for the statistical analysis of the collective excitations in cold deformed nuclei. In addition, we point out that, according to discussions in Ref. [10], the choice of an unfolding procedure does not influence much on the short-range spectral statistics in terms of the NNSDs calculated here.

The NNSD obtained in such a way are normalized to one. Then, they are fitted by simple theoretical distributions.

\section{WIGNER-DYSON NNSD}

\section{A. General ingredients}

Following the review [18], one obtains the probability $p(s)$ of finding the spacing $s$ between the nearest neighboring levels, (Eq. (11) and Refs. [6, 7, 12, 16]),

$$
p(s)=\aleph^{-1} g(s) \exp \left(-\int_{0}^{s} g\left(s^{\prime}\right) \mathrm{d} s^{\prime}\right) .
$$

As mentioned in Introduction, the key quantity $g(s)$ is the level repulsion density, $g(s)=\mathrm{d} N / \mathrm{d} s$, where $\mathrm{d} N$ is the number of states in the interval $\mathrm{d} s$ from $s$ to $s+\mathrm{d} s$ (see Appendix). It is convenient to consider $s$ in units of the average $D$ of distances between levels, $s=S / D$, where $S$ is the energy spacing, i.e., the distance between the neighbor levels in usual energy units. Thus, $D$ is locally a mean distance between neighboring levels in energy units.

Practically, the normalization factor $\aleph$ of the probability distribution (6) can be found with any accuracy for a large maximal value of $s, s_{\max }$,

$$
\aleph=\int_{0}^{s_{\max }} \mathrm{d} s g(s) \exp \left(-\int_{0}^{s} g\left(s^{\prime}\right) \mathrm{d} s^{\prime}\right) .
$$

This normalization factor is relatively obtained from the normalization condition at $s_{\max }$ going to $\infty$ :

$$
\int_{0}^{s_{\max }} p(s) \mathrm{d} s=1 .
$$

Another normalization condition writes

$$
\int_{0}^{s_{\max }} s p(s) \mathrm{d} s=1 .
$$

It is convenient to keep formally the upper integration limit $s_{\max }$ as a large finite number by reasons explained below. Notice that, also for convenience, we introduced the dimensionless quantities, such as the probability distribution $p(s)$, and the level repulsion density $g(s)$ as functions of the dimensionless spacing variable $s$ [in Ref. [18], the probability density is denoted by $P(S)$, where $S=s D$, and the level repulsion density - by $\left.r_{10}(S)\right]$.

With the definition of the dimensionless density $g(s)$, for the uniform case one has $g(s)=1$. This corresponds, in usual energy units, to the energy density $1 / D$. Substituting this constant level density $g(s)$ into Eq. (6), one has the Poisson law

$$
p_{\mathrm{P}}(s)=\exp (-s)
$$

The Wigner law follows from the assumption of the level repulsion density that is proportional to $s$. In this case, from Eq. (6) one finds

$$
p_{\mathrm{W}}(s)=(\pi s / 2) \exp \left(-\pi s^{2} / 4\right) .
$$

Both distributions are normalized to one for a large maximal value of $s$ in order to satisfy Eqs. (8) and (9) at large $s_{\max }$ and, precisely, at $s_{\max } \rightarrow \infty$.

The density $g(s)$ in fact is not a constant or simply proportional to $s$. A simple distribution based on the two-parameter linear approximation to the level repulsion density $g(s)$, that bridges the both Poisson (10) and Wigner (11) limits, will be considered first in the next section.

\section{B. A linear level-repulsion density approximation}

Keeping a link with the analytical properties of the level repulsion density $g(s)$ (Appendix), it is convenient to define the probability $p(s)$ [Eq. (6)] for a general smooth density $g(s)$ as a polynomial of not too a large power. As shown in Appendix, it is important to care of this density smoothness. For the simplest statistical analysis in terms of the Poisson- and Wigner-like distribution contributions, one can use the linear approximation of $g(s)$ in terms of the two free parameters $a$ and b,

$$
g(s)=a+b s
$$


Substituting Eq. (12) into the general Wigner-Dyson formula (6) and using the normalization condition (8) with large but a finite upper limit $s_{\max }$ (larger than the experimental data), one obtains explicitly the analytically simple LWD approximation [24]:

$$
p(s)=\frac{1+b s / a}{\aleph_{0}+b \aleph_{1} / a} \exp \left(-\frac{b}{2} s^{2}-a s\right),
$$

where

$$
\begin{aligned}
\aleph_{0} & =\int_{0}^{c} \mathrm{~d} s \exp \left(-\frac{b}{2} s^{2}-a s\right) \\
& =\sqrt{\frac{\pi}{2 b}} \exp \left(\frac{a^{2}}{2 b^{2}}\right)\left[\operatorname{erf}\left(\frac{a+b c}{\sqrt{2 b}}\right)-\operatorname{erf}\left(\frac{a}{\sqrt{2 b}}\right)\right], \\
\aleph_{1} & =\int_{0}^{c} \mathrm{~d} s s \exp \left(-\frac{b}{2} s^{2}-a s\right) \\
& =\frac{1}{b}\left[1-\exp \left(-\frac{b}{2} c^{2}-a c\right)-a \aleph_{0}\right]
\end{aligned}
$$

with $c=s_{\max }=S_{\max } / D$ being the maximal value of $s$. Then, we should check the second normalization condition (9) by choosing the parameter $c$ larger than all of the experimental NNSD spacings $s$. In practice, it is convenient to perform the three-parameter fitting over parameters $a, b$, and $c$ to the experimental NNSD provided that the normalization condition (9) is satisfied, and then, check that $c$ is sufficiently large with a good accuracy. In the limit $c \rightarrow \infty$, one has simply

$$
\aleph_{0} \rightarrow \sqrt{\frac{\pi}{2 b}} \exp \left[\frac{a^{2}}{2 b^{2}}\right], \quad \aleph_{1} \rightarrow \frac{1}{b}\left[1-a \sqrt{\frac{\pi}{2 b}}\right] .
$$

Taking the limits $a \rightarrow 1, b \rightarrow 0$ and $a \rightarrow 0, b \rightarrow \pi / 2$ in Eq. (13), one simply arrives relatively at the standard Poisson $g_{P}(s)$, Eq. (10), and Wigner $g_{W}(s)$, Eq. (11), distributions. In this way, a linear approximation (12) unifies analytically these two limit cases through a smooth level-repulsion density $g(s)$. Its parameters $a$ and $b$ in Eq. (12) (after their normalization to one for convenience) measure the probability to have separately the Poisson and Wigner distribution contributions.

\section{The Brody distribution}

The Brody distribution can be derived analytically from Eq. (6) by assuming the following expression for the level repulsion density:

$$
g(s)=\alpha s^{q} .
$$

With the normalization condition (8), another condition (9) is satisfied identically. Finally, one finds [10, 13, 18]

$$
p_{\mathrm{B}}(s)=\alpha(q) s^{q} \exp \left[-\frac{\alpha(q)}{q+1} s^{q+1}\right],
$$

where

$$
\alpha=(1+q)\left[\Gamma\left(\frac{q+2}{q+1}\right)\right]^{q+1} .
$$

Here, $\Gamma(x)$ is the standard Gamma function, and $q$ is a free parameter. The values $q=0(s>0)$ and $q=1(s \geq$ 0 ) in Eq. (17) correspond to the same Poisson [Eq. (10)] and Wigner [Eq. (11)] distributions.

The only one parameter is an advantage of the popular distribution $g_{B}(s)$ [Eq. [17)], suggested by Brody, over the approximation (13) based on the linear levelrepulsion density $g(s)$. As compared to the Brody approach, the two-parameter LWD approximation (13) is, to some extent, more general and better founded within the WD analysis in terms of the ordered Poisson and chaotized Wigner distributions. As a linear approximation (13) for $g(s)$, it has a more clear meaning of the intermediate values of the parameters, found from the least-square fitting to the experimental NNSD. In this way, one obtains the separate Poisson and Wigner distribution contributions. This is in addition to the Brody distribution (17) based on the power density (16). Such a density [Eq. (16)] does not satisfy a smoothness property of the level repulsion densities $g(s)$, in spite of using it in derivations of the NNSD within the WD theory (see Appendix). However, as shown below the results obtained by the LWD and the Brody approach are largely well agreed to each other.

Thus, the probability density (13) is a simple analytical continuation from the Poisson $g_{P}(s)$ to Wigner $g_{W}(s)$ limit distributions through a smooth linear levelrepulsion density $g(s)$. For a comparison and completeness, the statistical analysis of the experimentally obtained excitation-energy distributions are performed below within both the LWD and Brody approximations.

\section{Cumulative NNSD}

To complement our NNSD analysis of nuclear spectra, one can use the cumulative NNSDs. The cumulative NNSD is used as an alternative method to study the statistical properties of the experimental cumulative NNSD depending on the spacing variable $s$, in addition to the NNSD [10, 19]. In this subsection, we restrict ourselves to the additional information about the nuclear level statistics, depending on the spacing variable $s$, from the cumulative spacing distributions to support our NNSD results. A more proper quantitative study of these statistical properties of nuclear excitations will be in forthcoming work.

Let us consider the cumulative nearest-neighbor spacing distribution,

$$
F(s)=\int_{0}^{s} p\left(s^{\prime}\right) \mathrm{d} s^{\prime} .
$$

This integral distribution is the probability to find the spacing $s^{\prime}$ between the two-neighbor energy levels smaller or equal to a given value of $s$. For the cumulative Poisson distribution, one can explicitly obtain from Eqs. (19) and (10)

$$
F_{\mathrm{P}}(s)=1-\exp (-s) .
$$

For the corresponding Wigner distribution limit of $F(s)$, 
one finds

$$
F_{\mathrm{W}}(s)=1-\exp \left(-\pi s^{2} / 4\right) .
$$

Analytical expressions for the cumulative distributions obtained easy from the LWD [Eq. (13)] and the Brody [Eq. (17)] approach to the probability density will be worked out later.

\section{DISCUSSIONS OF THE RESULTS}

Experimental nearest-neighbor spacing distributions fitted by the LWD approximation [Eq. (13)] and the Brody approach [Eq. (17)] are presented in Figs. 3 5. Parameters of fittings are given in Table [I] The sampling interval $\gamma_{s}=0.2$, used for building the experimental NNSD (Sec. III), is taken from the condition of the stable smoothed NNSD values without sharp jumps between the neighbor data. This is similar to the so-called plateau condition in the smoothing procedure for calculations of the averaged level density [42, 43]. The plateau condition means the independence of the averaging parameters. As follows from Table [II the normalization condition (9) is satisfied in our calculations with good accuracy at $c \gtrsim 10$, that is significantly larger than any of energy intervals found from experimental data.

To build the NNSD for the rare-earth nuclei (Sec. III), the experimental $0^{+}$state energies limited by the $3 \mathrm{MeV}$ excitation are used for $\left.{ }^{158} \mathrm{Gd}[31],{ }^{168} \mathrm{Er} 32\right],{ }^{152,154} \mathrm{Gd}$, ${ }^{162} \mathrm{Dy},{ }^{168} \mathrm{Er},{ }^{176} \mathrm{Hf},{ }^{180,184} \mathrm{~W}$, and ${ }^{190} \mathrm{Os} \mathrm{33}$ ], $\left.{ }^{170} \mathrm{Yb} 34\right]$ nuclei [Fig. 3(a)]. The experimental NNSD for nuclei ${ }^{158} \mathrm{Gd}\left[32\right.$ and ${ }^{168} \mathrm{Er}$ [35] [Fig. 3(b)] is a special case since only for these two nuclei the measurements were carried out for larger excitation energies up to $4.2 \mathrm{MeV}$. The results of fitting are following. The rare-earth nuclear spectrum is described by $39 \%$ of the Poisson- and $61 \%$ of the Wigner-distribution contribution. They correspond approximately to the parameter $\mathrm{q}=0.48$ in the Brody approach. Simultaneously, for the ${ }^{158} \mathrm{Gd}$ and ${ }^{168} \mathrm{Er} \mathrm{cou}-$ ple, these parameters are given by $76 \%$ and $24 \%$, respectively, in the LWD approximation. This can be tentatively related to the value $\mathrm{q}=0.20$ for the Brody distribution. This means that the experimental $0^{+}$spectra in the energy interval $0-3 \mathrm{MeV}$ are intermediate between an order and a little more pronounced chaos structure, while the ordered nature is dominant for the experimental spectra in the energy interval about $0-4 \mathrm{MeV}$.

As was pointed above (Sec. IIA), the experimental NNSDs for actinide nuclei are available also for $2^{+}, 4^{+}$ and $6^{+}$collective states, along with $0^{+}$excitations. Long sequences of $2^{+}, 4^{+}$, and even $6^{+}$states, as well as $0^{+}$ states, all identified in ${ }^{228} \mathrm{Th}[28],{ }^{230} \mathrm{Th}[27],{ }^{232} \mathrm{U}$ [29] and ${ }^{242} \mathrm{Pu}$ [30, 36], are used in our analysis. As seen from Table II, the picture is similar to the rare-earth behavior. All spectra in the same energy interval 0-3 $\mathrm{MeV}$ demonstrate an intermediate structure between an order and a chaos structure with varying dominance of the Wigner to the Poisson contribution for increasing the angular momentum up to $4^{+}$. If for $0^{+}$states the Wigner contribution dominates with $79 \%(q=0.58)$, for the states with higher angular momenta, namely, for the $2^{+}$and $4^{+}$states, the Wigner contribution is somehow lowering. For example, one can conclude that the fluctuation properties for the $2^{+}$states is closer to the Poisson distribution than those for the $0^{+}$states. See Sec. IIA for discussions of the level sequence completeness. For the $4^{+}$states the Poisson contribution becomes even dominating with $62 \%(q=0.28)$.

The results for $6^{+}$states seem to be different from this trend. The $6^{+}$NNSDs are also more close to the Possion distribution as compared to $0^{+}$ones while the opposite tendency takes place with respect to the $4^{+}$ states: The Poisson contribution for $6^{+}$states is found less than that for $4^{+}$states. As was already pointed out in Sec. IIA the sequence for the $6^{+}$levels can not be apparently reliably completed because of a sharp drop in the cross-section value with increasing the angular momentum. This can be a reason of missing levels and, therefore, of their NNSD deflection from a general trend (see Sec. IIA for more details). A further progress in experimental studies of such high-spin collective states can be apparently helpful to clarify more the $6^{+}$statistical properties.

An increase of the Poisson distribution contribution with the nuclear spin value can be considered, to some extent, as that with a growth of the energy: The array of the states with higher angular momenta is shifted to larger energy excitations, too, as compared to the $0^{+}$ case. This behavior of the statistical distribution would look strange when accepting that increasing the excitation energy means an increase of the temperature, or of the thermodynamic entropy. Such an entropy production could be interpreted as a growing chaos. This would mean that the Wigner distribution contribution should be greater for higher energies. However, as it was emphasized above, one can conclude about the collective nature of states excited in the $(p, t)$ reaction, see Refs. 27, 37]. Collective excitations under the consideration in deformed nuclei can not be associated with an increase of temperature. Our results certainly different from those obtained [23] for the complete sets of noncollective states in nucleus ${ }^{208} \mathrm{~Pb}$. For such states, the chaoticity dominates both in the experimental and spectrum and that calculated within the shell model. Our calculations are in accordance with the results of Shriner at al. [19] and discussions in Ref. [10]. In any case, our statistical analysis provides another view on developments of a more microscopic model for the theoretical calculations of the collective modes.

Following the ideas of Refs. [19, 23] we tested the validity and completeness of the level sequences by comparing the experimental data with the collective state spectra calculated within the quasiparticle phonon model [32, 37]. Fig. [5 presents the distributions for $0^{+}$states in three actinides ${ }^{228,230} \mathrm{Th}$ and ${ }^{232} \mathrm{U}$. The experimental NNSD in the region of $0-3 \mathrm{MeV}$ (a) are compared with the two theoretical distributions. One of them is given in the same energy region (b) and another distribution - in the extended energy interval $0-4 \mathrm{MeV}$ (c). The parameters for the distributions shown on the panels (a) and (b) are approximately the same within the error limit accuracy. This agreement between the experimental and theoretical results confirms the collective nature of the 


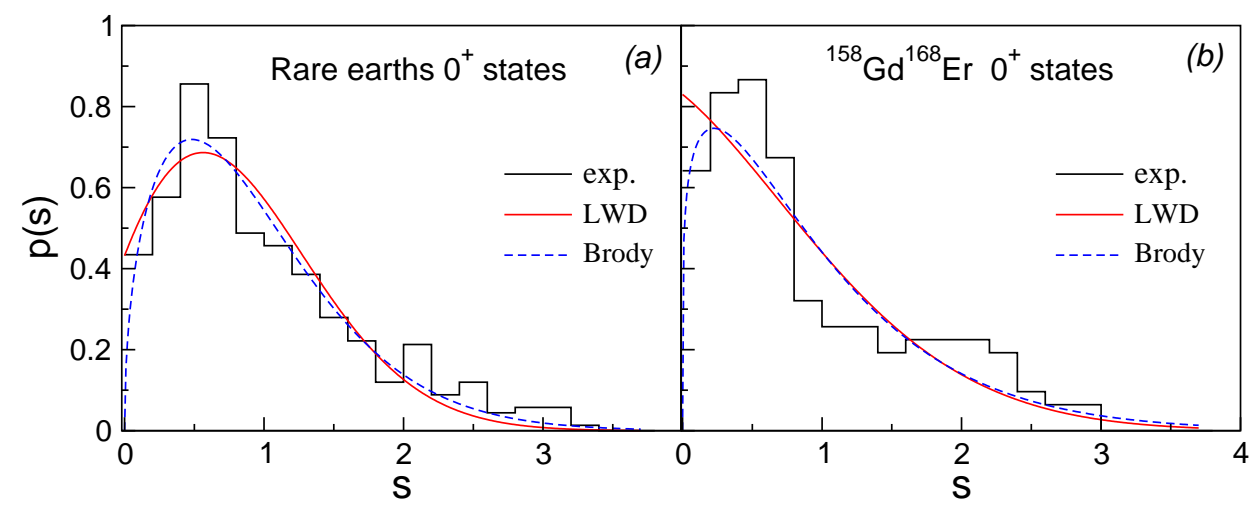

FIG. 3. Nearest neighbor spacing distributions $p(s)$ as functions of a dimensionless spacing variable $s$ for $0^{+}$states in the rare-earth nuclei and fits by the LWD approximation (red solid lines) and the Brody approach (blue dashed lines); (a): for a number of the rare-earth nuclei up to the energy $3 \mathrm{MeV}$ (see the text); (b): for the ${ }^{158} \mathrm{Gd}$ and ${ }^{168}$ Er nuclei up to about 4 $\mathrm{MeV}$. A sampling interval of $\gamma_{s}=0.2$ was used.

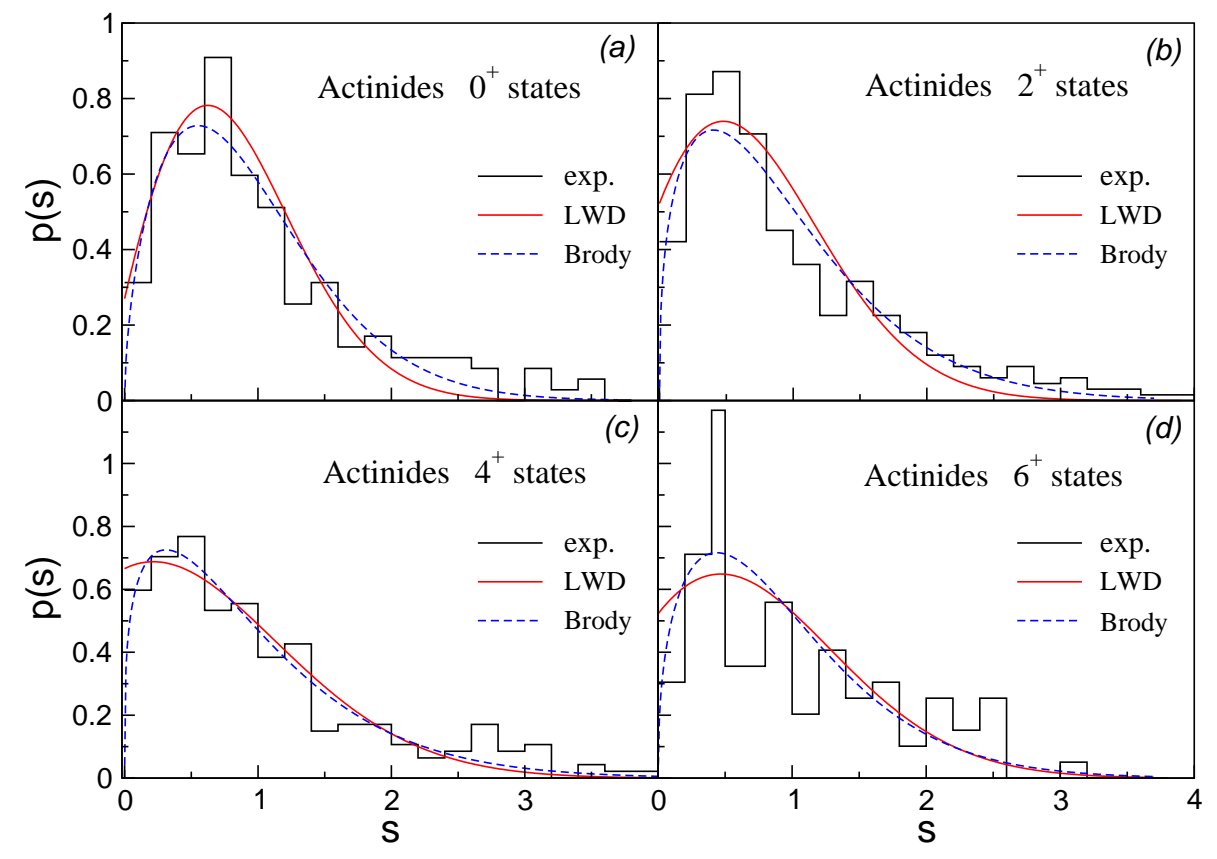

FIG. 4. The same as in Fig. 3 but for different states in the actinide nuclei; (a-d): for $0^{+}, 2^{+}, 4^{+}$, and $6^{+}$states, respectively. The same fits by the LWD (red solid lines) and the Brody (blue dashed lines) approach as in Fig. 3 are shown.

$0^{+}$states and, finally, the completeness of the level sequences. At the same time, the theoretical distribution for the energy interval $0-4 \mathrm{MeV}$ [Fig. [5(c)] is shifted to the Poisson law as compared to the experimental and theoretical distributions in the interval $0-3 \mathrm{MeV}$. It is in agreement with the results obtained for the ${ }^{158} \mathrm{Gd}$ and ${ }^{168}$ Er nuclei (see Fig. 3(b)).

Fig. 6] shows the cumulative distributions $F(s)$ [Eq. (19)] for the $0^{+}(\mathrm{a}), 2^{+}$(b), $4^{+}$(c), and $6^{+}$(d) states excited in the same actinide nuclei as in Fig. 4.
The dotted lines represent the corresponding cumulative Poisson distribution [Eq. (20)]. All dashed lines in Fig. 6 show the Wigner distribution limit of $F(s)$ [Eq. (21)]. As seen from this Figure, for all $0^{+}, 2^{+}, 4^{+}$and $6^{+}$states the Wigner cumulative distribution (21) well reproduces the behavior of empirical distributions $F(s)$ [Eq. (19)] at small and intermediate spacings $s$. On the other hand, at large spacings, $F(s)$ approaches basically the Poisson cumulative-NNSD limit (20). Such a peculiarity of a cumulative distribution implies a chaotic arrangements 


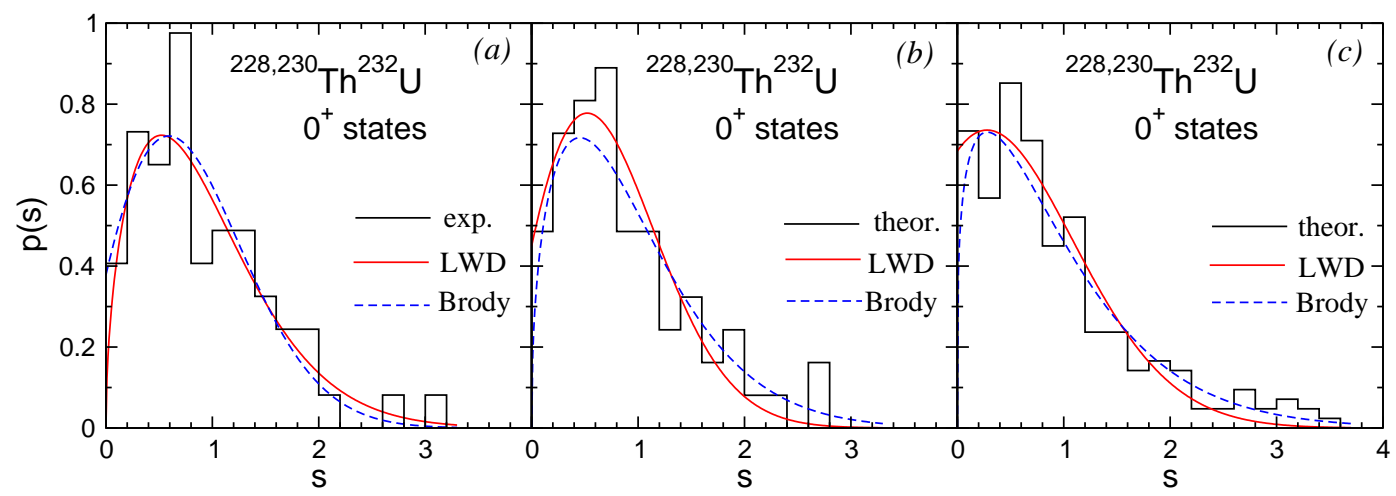

FIG. 5. Comparison of the NNSD between the experimental data $(a)$ and the theoretical quasiparticle-phonon model results (b) in the energy interval up to $3 \mathrm{MeV}$ in the ${ }^{228,230} \mathrm{Th}$ and ${ }^{232} \mathrm{U}$ actinide nuclei, and those $(c)$ - up to $4 \mathrm{MeV}$. Other notations are the same as in Figs. 3 and 4

\begin{tabular}{|c|c|c|c|c|c|c|c|c|}
\hline Figs. & Nuclei & States & $\mathrm{a}(\%)$ & $\mathrm{b}(\%)$ & $\int s p(s) \mathrm{d} s$ & Accuracy & $q$ & Accuracy \\
\hline $3 a$ & Rare earths & $0^{+}$exp. & $0.43(39)$ & $0.69(61)$ & 1.04 & 8.1 & 0.48 & 6.7 \\
\hline $3 \mathrm{~b}$ & ${ }^{158} \mathrm{Gd}{ }^{168} \mathrm{Er}$ & $0^{+}$exp. & $0.83(76)$ & $0.26(24)$ & 0.96 & 11.3 & 0.20 & 10.0 \\
\hline $4 a$ & Actinides & $0^{+}$exp. & $0.27(21)$ & $1.01(79)$ & 1.02 & 9.2 & 0.58 & 8.9 \\
\hline $4 b$ & & $2^{+}$exp. & $0.52(41)$ & $0.75(59)$ & 0.95 & 10.2 & 0.38 & 8.2 \\
\hline $4 c$ & & $4^{+}$exp. & $0.67(62)$ & $0.41(38)$ & 1.00 & 8.5 & 0.28 & 7.3 \\
\hline $4 \mathrm{~d}$ & & $6^{+}$exp. & $0.52(50)$ & $0.52(50)$ & 1.06 & 14.9 & 0.42 & 13.7 \\
\hline $5 \mathrm{a}$ & ${ }^{228,230} \mathrm{Th},{ }^{232} \mathrm{U}$ & $0^{+}$exp. & $0.38(32)$ & $0.80(68)$ & 1.03 & 10.5 & 0.54 & 10.1 \\
\hline $5 b$ & & $0^{+}$theor. & $0.45(33)$ & $0.91(67)$ & 0.94 & 9.7 & 0.44 & 9.5 \\
\hline $5 \mathrm{c}$ & & $0^{+}$theor. & $0.68(56)$ & $0.54(44)$ & 0.93 & 8.7 & 0.25 & 8.9 \\
\hline
\end{tabular}

TABLE II. Parameters $a$ and $b$ of the LWD and $q$ of the Brody approximation for the collective excited states in several nuclei. The 1st column refers to the corresponding figures 35 The Poisson and Wigner contributions are given also as $a$ and $b$ normalized to $100 \%$ in circle brackets in 4 th and 5 th columns, respectively. The normalization integral of Eq. (9) at these $a, b$ and $c \gtrsim 10$ is given too in 6th column. The accuracy $\left(\chi^{2}\right)$ of the least-square fitting (in percents) are shown, respectively, in the 7th and 9th columns for the LWD and Brody calculations.

of close-lying levels and regular ones of the significantly separated levels. This is in agreement with our results for the NNSD plotted in Figs. 3.5. As in the case of using the NNSD (see Fig. 4), the cumulative distribution analysis of Fig. [6] shows that the relative Poisson contribution (20) grows with the increase of the spin of nuclear states.

\section{CONCLUSIONS}

We provide the statistical analysis of the collective excitations with several spins: $0^{+}$in a number of the rareearth nuclei; and $0^{+}, 2^{+}, 4^{+}$, and $6^{+}$in a few actinide nuclei by using the simple approximations to the WignerDyson probability distribution. These approximations to the nearest neighbor spacing distribution are based on different properties of the level repulsion density. For the linear approximation to this density, one obtains a clear information on the quantitative measure of the Poisson order and Wigner chaos contributions in the experimental data, separately, in contrast to the heuristic Brody approach. However, one finds in our calculations that the Brody formula [Eq. (17)] agrees largely well with the LWD probability-distribution results [Eq. (13)].

We found the intermediate structure between the Poisson and Wigner statistical peculiarities of the experimental spectra by evaluating their separate contributions. The NNSD for a smaller excitation-energy region can be described better by the Wigner distribution. The NNSD for an extended interval of the collective excitations, including higher energies, becomes more close to the Poisson distribution. Also, one finds that the Wigner contribution dominates in the NNSD for $0^{+}$states and the Poisson contribution is larger with increasing the angular momentum. This looks in line of the adiabatic picture for different collective-excitation modes in deformed nuclei.

The experimental NNSDs are in agreement with the theoretical calculations for the same energy interval within the quasiparticle phonon model, that confirms the completeness of the used spectra. The comparison of these results with the theoretical ones for larger energy interval supports the same conclusion about a shift 


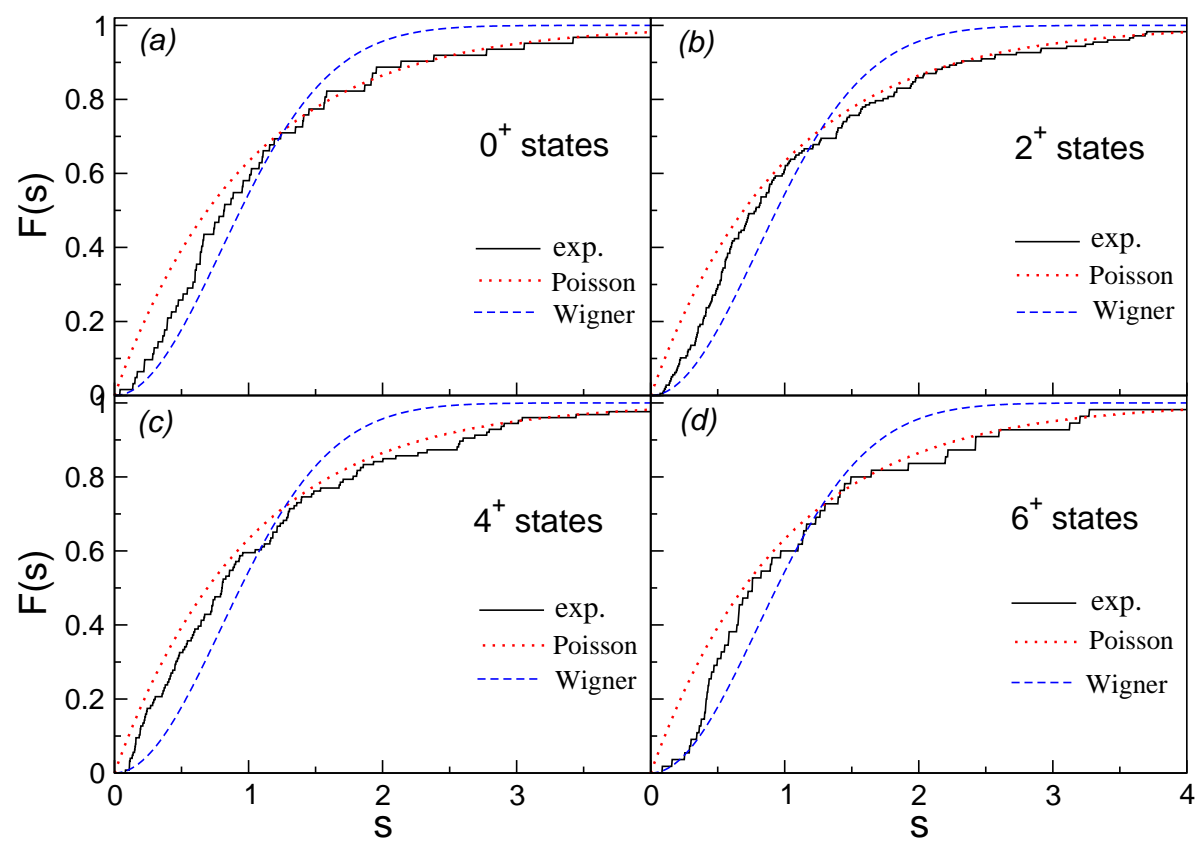

FIG. 6. Histograms of the cumulative nearest-neighbor spacing distributions (19) for the $0^{+}$(a), $2^{+}$(b), $4^{+}(\mathrm{c})$ and $6^{+}(\mathrm{d})$ states in the actinide nuclei discussed in Fig. 4. Dotted and dashed lines are the Poisson distribution [Eq. (20)] and the Wigner distribution [Eq. (21)] limit of these cumulative distributions (19), respectively.

from the Wigner to the Poisson contribution dominance. As emphasized in Ref. [10], for the collective states in deformed nuclei the statistical distributions are closer to the Poisson distribution, and in other cases the situation is intermediate (see also Ref. 19]). This picture looks in agreement with our statistical results for the collective states.

With the help of the cumulative distributions, for the $0^{+}, 2^{+}, 4^{+}$and $6^{+}$states in actinide nuclei we show that the chaotic cumulative Wigner limit well reproduces the behavior of empirical cumulative distributions $F(s)$ at small and intermediate spacings $s$. At larger spacings they approach the regular Poisson cumulativedistribution limit. In line of the nearest-neighbor spacing distribution calculations, the cumulative distribution analysis shows also that the relative Poisson contribution grows with the increase of the spin of nuclear states.

As perspectives, we are also going to study more the Wigner-Dyson probability-density approach within simple approximations and apply them more systematically to learn the statistical properties of experimental data. In this way, it will be worth to calculate the nearest neighbor spacing distributions for a nonlinear levelrepulsion density to describe other statistically observable spectra of the collective nature beyond the Wigner and Poisson contributions. We are going also to understand the influence of the symmetry breaking phenomena on these distributions of the collective states in deformed nuclei.

\section{ACKNOWLEDGEMENTS}

We are grateful to M. Spieker for providing us with experimental results before publication, also to K. Arita, S. Aberg, J. Blocki, V. M. Kolomietz, S. Mizutori, and K. Matsuyanagi for many helpful discussions. One of us (A.G.M.) is also very grateful for kind hospitality during his working visits of Physical Department of the Nagoya Institute of Technology, also the Japanese Society of Promotion of Sciences for financial support, Grant No. S14130.

\section{Appendix: The derivation of the NNSD}

We introduce first the level repulsion density, $g(s)$, as the number of the levels $\mathrm{d} N$ in the dimensionless energy interval $[e+s, e+s+\mathrm{d} S]$, divided by the energy interval length $\mathrm{d} s, g(s)=\mathrm{d} N / \mathrm{d} s$ [18]. With the help of this quantity, one can derive the NNSD $p(s)$ as the probability density that is a function of the spacing $s$ between the nearest neighboring levels in dimensionless units $e=E / D$ and $s=S / D$, where $D$ is locally the averaged distance between neighbor levels. Specifying $p(s)$ to the problem with the known spectra of the manybody (or single-particle) Hamiltonian, one can split relatively a small energy interval $\Delta e$ under the investigation into many small (equivalent for simplicity) parts $\gamma_{s} \ll \Delta e$. Each of $\gamma_{s}$, nevertheless, contain many energy levels. Then, we find the number of the levels which occur inside of the relatively small interval $\gamma_{s}$. Normalizing these numbers by the total number of the levels inside the total energy interval $\Delta e$, one obtains the distribution which we shall call as the probability density $p(s)$. 
Notice that the result of this calculation depends on the spacing length of the selected $\gamma_{s}$. In our calculations, we select $\gamma_{s}$ by the condition of a sufficient smoothness of the distribution $p(s)$. We have to study $p(s)$ as a function of $\gamma_{s}$ at a given $s$ for several values of the parameters of this distribution to find a so called "plateau" in $\gamma_{s}$, i.e., a region of $\gamma_{s}$ values where $p(s)$ can be approximately considered as a constant independent of $\gamma_{s}$ and above mentioned parameters (see Refs. [42, 43]). Such a procedure is often used for the statistical treatment of the experimentally obtained spectrum with the fixed quantum numbers as the angular momentum, parity and so on [18] (Sec. II).

Following mainly Ref. 7], let us calculate first the intermediate quantity $f(s)$ as the probability that there is no an energy level in the energy interval $[e, e+s]$. According to a general definition of the level repulsion density mentioned above, $g(s) \mathrm{d} s$, can be considered as the probability that there is one energy level in the interval $[e+s, e+s+\mathrm{d} s]$. Then, one has

$$
f(s+\mathrm{d} s)=f(s)(1-g(s) \mathrm{d} s)
$$

Assuming that $f(s)$ is a smooth function of $s$, one can expand $f(s+\mathrm{d} s)$ with respect to $\mathrm{d} s$. Thus, the relationship (A1) leads to the differential equation for $f(s)$,

$$
\mathrm{d} f=-g(s) \mathrm{d} s f(s) .
$$

Solving this equation, one gets

$$
f(s)=C \exp \left(-\int_{0}^{s} g\left(s^{\prime}\right) \mathrm{d} s^{\prime}\right),
$$

where $C$ is arbitrary unknown constant. Note that the assumption that $f(s)$ is a smooth function of $s$ can be satisfied if $g(s)$ is also a smooth function of $s$, i.e., the density $g(s)$ can be approximated by a polynomial in powers of $s$ of not too a high power. Notice also that a constant density, $g(s)=a$, and linear, $g(s)=b s$, functions of $s$, in which $a$ and $b$ are constants, obey this smoothness condition. They are related to the limit cases of the linear density $g(s)=a+b s$, namely, the Poisson (zero order polynomial, $b=0$ ) and the Wigner (first order polynomial with $a=0$ ) distribution functions. Let $p(s) \mathrm{d} s$ denotes the probability that the next energy level occurs in the interval $[e+s, e+s+\mathrm{d} s]$,

$$
p(s) \mathrm{d} s=f(s) g(s) \mathrm{d} s .
$$

Then, substituting Eq. (A3) into Eq. (A4), one finally arrives at the general distribution:

$$
p(s)=C g(s) \exp \left(-\int_{0}^{s} g\left(s^{\prime}\right) \mathrm{d} s^{\prime}\right) .
$$

The boundary conditions in solving the differential equation (A2) accounts for the meaning of the NNSD $p(s)$ and its argument as the spacing between the nearest neighbor levels as shown in the integration limit in Eq. (A5). The constant $C$ is determined by the normalization condition (8) [see Eq. (7)]. We have also to care of another normalization condition (9) for using the correct $D$ units.
[1] F. Iachello and A. Arima, The Interacting Boson Model (Cambridge University Press, Cambridge, England, 1987).

[2] V. G. Soloviev, Theory of Atomic Nuclei: Quasiparticles and Phonons (Institute of Physics, Bristol, 1992).

[3] A. Bohr and B. R. Mottleson, Nuclear Structure: Nuclear Deformations, Vol. 2 (Benjamin, New York,1975).

[4] P. Ring and P. Schuck, The Nuclear Many-Body Problem (Springer-Verlag, New York, Heisenberg, Berlin, 1980).

[5] A.B. Migdal, Finite Fermi-system theory and properties of atomic nuclei (Moscow, Nauka, Fizikomatematicheskaya literatura, 1983).

[6] M. L. Mehta, Random Matrices (Academic Press, San Diego, New York, Boston, London, Sydney, Tokyo, Toronto, 1991).

[7] S. Aberg, Quantum Chaos (Mathematical Physics, Lund, 2002).

[8] H. A. Weidenmüller and G. E. Mitchell, Rev. Mod. Phys., 81, 539 (2009).

[9] G. E. Mitchell, A. Richter, H. A. Weidenmüller, Rev. Mod. Phys. 82, 2845 (2010).

[10] J. M. G. Gomez, K. Kar, V. K. B. Kota, R. A. Molina, A. Relano, and J. Retamosa, Phys. Rept. 499, 103 (2011).

[11] O. Bohigas, M. J. Giannoni, C. Schmit, Phys. Rev. Lett. 52, 1 (1984).

[12] E. P. Wigner, Proc. Philos. Soc., 47, 790 (1951).
[13] T. A. Brody, Lett. Nuovo Cimento 7, 482 (1973).

[14] M. V. Berry and M. Robnik, J. Phys. A 17, 2413 (1984).

[15] F. M. Izrailev, Phys. Lett. A 134, 13 (1988).

[16] C. E. Porter, Statistical Theories of Spectra: Fluctuations (Academy Press, New York, 1965).

[17] M. V. Berry, Ann. Phys. (N.Y.), 131, 163 (1981).

[18] T. A. Brody, J. Flores, J. B. French, P. A. Mello, A. Pandey, and S. S. M. Wong, Rev. Mod., 53, 385 (1981).

[19] J. F. Shriner Jr., G. E. Mitchell, T. von Egidy, Z. Phys. A 338, 309 (1991).

[20] J. F. Shriner Jr., E .G. Bilpuch, P. M. Endt, G. E. Mitchell, Z. Phys. A 335, 393 (1990)

[21] J. F. Shriner Jr., C. A. Grossmann, and G. E. Mitchell, Phys. Rev. C 62, 054305 (2004).

[22] G. Vidmar, H.-J. Stökmann, M. Robnik, U. Kühl, R. Höhmann, S. Grossman, J. Phys. A 40, 13803 (2007)

[23] B. Dietz, A. Heusler, K. H. Maier, A. Richter, and B. A. Brown, Phys. Rev. Lett., 118, 012501 (2017); Phys. Rev. C 95, 14317 (2017).

[24] J. P. Blocki and A. G. Magner, Phys. Rev. C 85, 064311 (2012).

[25] O. Bohigas and M. P. Pato, Phys. Lett. B 595, 171 (2004).

[26] A. I. Levon, J. de Boer, G. Graw, R. Hertenberger, D. Hofer, J. Kvasil, A. Lösch, E. Müller-Zanotti, M. Würkner, H. Baltzer, V. Grafen, and C. Günther, 
Nucl. Phys. A 576, 267 (1994).

[27] A. I. Levon, G. Graw, Y. Eisermann, R. Hertenberger, J. Jolie, N. Yu. Shirikova, A. E. Stuchbery, A. V. Sushkov, P. G. Thirolf, H.-F. Wirth, N. V. Zamfir, Phys. Rev. C 79, 014318 (2009).

[28] A. I. Levon, G. Graw, R. Hertenberger, S. Pascu, P. G. Thirolf, H.-F. Wirth, P. Alexa. Phys. Rev. C 88, 014310 (2013).

[29] A. I. Levon, P. Alexa, G. Graw, R. Hertenberger, S. Pascu, P. G. Thirolf, and H.-F. Wirth, Phys. Rev. C 92, 064319 (2015).

[30] M. Spieker, D. Bucurescu, J. Endres, T. Faestermann, R. Hertenberger, S. Pascu, S. Skalacki, S. Weber, H.F. Wirth, N.V. Zamfir, and A. Zilges, Phys. Rev. C 88, 041303(R) (2013).

[31] S. R. Lesher, A. Aprahamian, L. Trache, A. OrosPeusquens, S. Deyliz, A. Gollwitzer, R. Hertenberger, B. D. Valnion, and G. Graw Phys. Rev. C 66, 051305R (2002).

[32] D. Bucurescu, G. Graw, R. Hertenberger, H.-F. Wirth, N. Lo Iudice, A. V. Sushkov, N. Yu. Shirikova, Y. Sun, T. Faestermann, R. Krucken, M. Mahgoub, J. Jolie, P. von Brentano, N. Braun, S. Heinze, O. Moller, D. Mucher, C. Scholl, R. F. Casten, and D. A. Meyer, Phys. Rev. C 73, 064309 (2006).

[33] D. A. Meyer, V. Wood, R. F. Casten, C. R. Fitzpatrick, G. Graw, D. Bucurescu, J. Jolie, P. von Brentano, R. Hertenberger, H.-F. Wirth, N. Braun, T. Faester- mann, S. Heinze, J. L. Jerke, R. Krucken, M. Mahgoub, O. Moller, D. Mucher, and C. Scholl, Phys. Rev. C 74, 044309 (2006).

[34] L. Bettermann, S. Heinze, J. Jolie, D. Mucher, O. Moller, C. Scholl, R. F. Casten, D. Meyer, G. Graw, R. Hertenberger, H.-F. Wirth, and D. Bucurescu, Phys. Rev. C 80, 044333 (2009).

[35] A. I. Levon et al., to be published.

[36] M. Spieker et al., private comminications (2017).

[37] N. Lo Iudice, A. V. Sushkov, and N. Yu. Shirikova, Phys. Rev. C72, 034303 (2005).

[38] Y. A. Akovali, Nucl. Data Sheets 69, 155 (1993).

[39] B. Ackermann, H. Baltzer, K. Freitag, C. Gunther, P. Herzog, J. Manns, U. Muller, R. Paulsen, P. Sevenich, T. Weber, B. Will, J. de Boer, G. Graw, A. I. Levon, M. Loewe, A. Losch, and E. Muller-Zanotti, Z. Phys. A 350, 13 (1994).

[40] O. Bohigas and M.-J. Giannoni, Lecture Notes in Physics 209, 1 (1984).

[41] A. Gilbert and A. G. W. Cameron, Can. J. Phys. 43, 1446 (1965).

[42] V. M. Strutinsky, Nucl. Phys. A 95, 420 (1967); 122, 1 (1968).

[43] M. Brack, J. Damgaard, A. S. Jensen, H. C. Pauli, V. M. Strutinsky, and C. Y. Wong, Rev. Mod. Phys. 44, 320 (1972). 